\title{
Un ricordo di Franco Fontana
}

Matteo Caroli - Paolo Boccardelli - Raffaele Oriani

Americo Cicchetti

Silvia Profili

Marco Recchioni

Luca Giustiniano - Enzo Peruffo - Luca Pirolo

Americo Cicchetti - Silvia Profili - Marco Recchioni

Luciano Fratocch

Paola Achard

Un ricordo di Franco

Luciano Fratocchi - Paola Achard - Carlo Sirianni

Entrasti da professore ordinario alla Luiss alla fine degli anni '80; una delle prime chiamate di punta dell'ambizioso programma di rafforzamento dell'università voluto dall'allora presidente Guido Carli.

Ti venne dato l'obiettivo di sviluppare il filone degli studi sulla gestione e l'organizzazione d'impresa. Hai fondato quella Scuola, riempiendola di allievi, molti dei quali sono oggi in ruoli importanti nella tua stessa università, o si fanno valere in Italia o allestero. Ti occupasti poi di sviluppare l'offerta formativa e il potenziamento della Facoltà di Economia. Gestitisti tu, dal lato accademico, l'operazione delle "borse Amici della Luiss", istituite per far crescere all'interno giovani di belle speranze che sarebbero poi potuti divenire accademici di "scuola" Luiss.

Laltro grande progetto in cui fosti coinvolto fu il lancio dell'offerta di master e corsi post-laurea; già da tempo, la Bocconi aveva lanciato con forza la sua "Scuola di direzione aziendale", e in Italia, la domanda stava per esplodere. Divenisti rapidamente il leader operativo della Scuola di Management, (che poi sarebbe divenuta Luiss Management e oggi si chiama Luiss Business School); nel 1990 lanciasti la prima edizione del "Master in Business Administration".

Eri in facoltà da relativamente poco tempo, quando la Luiss fu attraversata da cambiamenti significativi. Alcuni grandi nomi lasciarono; il rettore Scognamiglio per la politica; Mario di Lazzaro, Carlo Caramiello prematuramente scomparsi; il Presidente Guido Carli morì nel 1993. La nostra università era pronta alla sfida di divenire una delle prime in Italia. Sei stato il protagonista indiscusso di quella sfida che la Luiss ha vinto, compiendo la sua prima grande trasformazione positiva. Proprio in quegli anni, infatti, ti venne affidata la direzione della Scuola di Management e di lì a poco fosti nominato Preside. Ti trovasti, quindi a guidare da un lato, la Facoltà che già allora attraeva la netta maggioranza degli studenti. Dall'altro, la struttura che aveva il potenziale di divenire un importante amplificatore di programmi della Luiss nel mondo delle imprese, oltre che un forte generatore di valore economico, come infatti è poi diventata. Un carico di lavoro enorme. Lo hai assolto con risultati eccellenti; quei risultati che sono stati la base per il grande slancio che stiamo vivendo.

Hai avuto una visione culturale; culturale, prima che tecnicoamministrativa, della nostra Facoltà, oggi Dipartimento e della Business School. Hai ideato tutti i percorsi formativi e li hai saputi far evolvere, unendo la tua personale competenza alla capacità di recepire stimoli e indicazioni, soprattutto dal mondo delle imprese e delle istituzioni. Chi 
sinergie

Vol. 38, Issue 3, 2020

partecipò a quel lavoro, poté comprendere come è leader colui che unisce il guardare lontano con il saper compiere il lavoro quotidiano, occupandosi anche delle questioni minute, apparentemente banali.

Hai lavorato, lavorato, e ancora lavorato. Hai anche fatto lavorare molto chi ti stava intorno; ma anzitutto, lavoravi tu personalmente; fedele al principio che l'esempio viene dall'alto. Le luci della tua stanza in palazzina a viale Pola rimanevano accese fino a tarda sera e non era raro trovarti il sabato. tu eri il tuo lavoro e il tuo lavoro si modellava sul tuo modo di essere

Per il ruolo istituzionale che avevi, fosti tu a disegnare il primo sistema " $3+2$ " della facoltà di Economia; un'impostazione di grande successo come hanno dimostrato negli anni successivi i numeri delle domande di ammissione in continua crescita. Hai guidato l'ideazione e l'implementazione della grande articolazione dellofferta post-laurea che, passando attraverso ben tre diversi modelli istituzionali, è arrivata oggi al pieno riconoscimento internazionale.

Nel frattempo, hai anche presieduto il comitato tecnico-organizzatore che creò la facoltà di Economia presso l'Università di l'Aquila e ti sei impegnato per lo sviluppo della prestigiosa Facoltà di ingegneria dello stesso Ateneo. Tributi sentiti e importanti alla tua terra natia, ove fosti a lungo anche Presidente operativo della Cassa di Risparmio di L'Aquila.

Negli ultimi anni della tua gestione, hai accompagnato il complesso processo di internazionalizzazione della Luiss Guido Carli, che ha tra l'altro portato la Business School ad essere formalmente accreditata tra le migliori al mondo. Anche in questo caso, hai offerto una grande lezione umana e professionale: saper passare il testimone quando è necessario per il bene dell'istituzione. In quegli anni, hai avuto modo di compiere la tua ultima grande innovazione organizzativa: l'istituzione del Dipartimento di impresa e management. Fu tua l'intuizione della sua importanza per dare ulteriore spinta alla Luiss come università di eccellenza nella formazione e negli studi manageriali e aziendali. Fu tua la progettazione: gli obiettivi strategici, i contenuti dell'offerta, le aree di sviluppo.

E poi cè stato il tuo lavoro scientifico. Non è qui il caso di fare l'elenco dei tuoi numerosissimi contributi. Basti citare i due libri "sommi": "Il sistema organizzativo aziendale", e "Lo sviluppo del personale", con la loro visione seminale di strategia e organizzazione interdipendenti e funzionanti sulla base delle persone, delle loro competenze e del modo in cui sono gestite. Sei stato un riferimento in tre settori disciplinari: quello di "Economia e gestione delle imprese", quello di "Organizzazione aziendale" e quello di "Ingegneria economico-gestionale". Hai teorizzato l'interdisciplinarietà e l'hai anche messa in pratica; caso raro nell'Accademia.

Ma il ricordo che lasci va ben oltre quello che hai fatto; è altrettanto vivido per quello che fu tuo modo di essere; per la tua forte empatia con le persone, le più diverse e a prescindere dalla loro posizione. Unempatia, singolarmente unita ad una burberaggine a tratti proverbiale che ti rendeva davvero unico. Una capacità di comprendere gli individui, le loro potenzialità, come i loro limiti; di capire come valorizzare le prime e non curarsi troppo dei secondi. Con un principio sempre chiaro in testa: "non lasciare nessuno indietro"; premiare chi merita, ma considerare che 
in un certo modo, tutti meritano qualcosa, perché ognuno offre un suo contributo.

Hai avuto a lungo ruoli di vertice, anche in grandi imprese e Istituzioni; li hai sempre gestiti nel solo interesse dell'istituzione che te li aveva attribuiti; da "civil servant" come si usa dire. Se non altro, lo dimostra il fatto che quando lo hai lasciato, sei stato beneficiato, non di incarichi o prebende, ma di una diffusa e profonda gratitudine. Nel lungo tempo in cui sei rimasto alla guida della Luiss Business School e della Facoltà di Economia, quelli che erano vascelli sono diventate corazzate; la nostra Comunità è cresciuta nel numero e nella qualità ed è rimasta coesa.

Dunque, Franco, dirti "grazie" può sembrare banale. Ma qualcuno ha spiegato che la semplicità è il modo migliore con cui si esprimono le più complesse tra le complessità.

Grazie per quello che hai fatto e per l'insegnamento che hai dato non solo con le parole, ma soprattutto con il tuo fare. Grazie per esserti curato di ciascuno di noi; grazie per averci sgridato; a volte, anche mortificato, insegnandoci così a diventare noi stessi.

Grazie per essere stato una persona onesta, giusta e per bene, e un vincente, rimanendo una persona semplice.

Grazie per essere stato un Maestro.

Matteo Caroli, Paolo Boccardelli, Raffaele Oriani, Luca Giustiniano,

Enzo Peruffo, Luca Pirolo, Americo Cicchetti, Silvia Profili,

Marco Recchioni, Luciano Fratocchi, Paola Achard, Carlo Sirianni

LUISS Guido Carli - Roma - Italy

Università Cattolica del Sacro Cuore - Milano - Italy

Università Europea di Roma - Italy

Università dell'Aquila - Italy

Università di Salerno - Italy

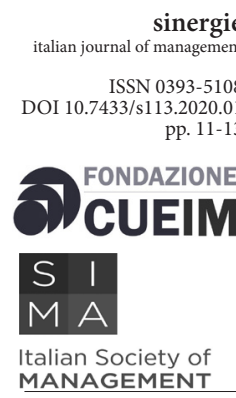

